\title{
Current and future use of virtual and augmented reality in minimal invasive oral surgery
}

\author{
Tortorici $\mathrm{S}^{1}$ and Difalco $\mathrm{P}^{2 *}$ \\ ${ }^{1}$ Professor of Oral Surgery, Department of Surgical, Oncological and Oral Sciences (Di.Chir.On.S.). University of Palermo; via del Vespro n.129, 90100 - Palermo, \\ Italy \\ ${ }^{2}$ Department of Surgical, Oncological and Oral Sciences (Di.Chir.On.S.). University of Palermo; via del Vespro n.129, 90100 - Palermo, Italy
}

In the last years there has been a change from the concept of traditionally oral surgery into minimal invasive surgery. Thus, a large number of practitioners started using magnification devices in their practice. These devices enhance visibility of the working field and permit to limit the size and number of cuts, or incisions. The most common are loupes, but they have limitations such as convergent vision, deficient magnification, image distortion, or small depth of focus. These limitations were superseded by the use of surgical microscope (SM) that allows amplification of detail and excellent visualisation of the working field. The SM is very important in the development of minimally invasive oral surgery, but today, the computer and new software play a key role. The main tissue types, organs and lesions can be identified, using medical imaging techniques, such as computed tomography (CT) or magnetic resonance imaging (MRI). Although these images contain all required information about patients and their organs, it may be difficult to perceive the extent of a lesion and its relationship with the surrounding tissues. Thus, the role of software for 3-dimensional (3D) visualization is very important. These software permit to visualize 3D images from a set of 2-dimensional (2D) images, using direct volume rendering technique; therefore, all acquired data can be composed to a digital 3D virtual model of the patient's mouth. According to its definition "Virtual reality (VR) refers to computergenerated environments or realities that are designed to simulate a person's physical presence in a specific environment that is designed to feel real". Using VR, an oral surgeon can visualize the lesions, to study a treatment plan, and can identify possible complications at the same time. Vision and tactile sense are the principal sensory inputs employed by humans in object manipulation tasks. Using VR and haptic technology, an oral surgeon will be able to simulate a virtual surgical treatment. The word "haptics" comes from the greek words haptikos (meaning able to come into contact with) and haptesthai (meaning touch or contact) ${ }^{1}$. Haptic technology, also known as kinaesthetic communication or 3D touch, is currently used in robot-assisted minimally invasive surgery [1]. The haptic interface is a device that allows a virtual or remote environment to stimulate the sense of touch on a human operator. Today, a number of haptic interfaces with different structures have been developed and are commercially available. These devices have been widely used in a multitude of applications such as graphical user interfaces, games, education and training etc. Using VR and haptic interfaces it will be possible create virtual patients and will be possible, also, to carry out a virtual oral surgical procedure. In surgical simulation, realistic interactions between surgeon, objects and virtual patient are very important, thus the end-effector of interfaces for surgical use, covers the natural range of motion of the human hand. Currently, these simulators are an important part of training of students and surgeons [2], but in the future it will be possible to realize a haptic hologram of each patient. A hologram is a three-dimensional image, created with photographic projection, using a tactile interface this image can become tangible. These haptic holograms will simulate the patient's tissues characteristics and it will permit the reproduction of lesions. Using haptic hologram surgeons will be able to simulate each oral intervention. After this virtual study of lesions, in the future, it will be possible to create a surgical guide based on augmented reality (AR). Although AR is a form of VR, it is different. The AR allows to the surgeon to overlay virtual objects in a real-world environment and mixed them. Now, this is possible, using a powerful computer with a dedicated software, a tracking device and a display. Tracking devices are used for registration. The registration is the process in which virtual objects, generated by a computer, are merged into the real-world image. Tracking device is an instrument or a pointer to establish correspondence between features in the preoperative images and the surgical scene. The most commonly used tracking devices in oral surgery is optical or mechanical. Today, the AR is particularly useful for the placement of endosseous dental implants. This dental application of AR is named dynamic guided implant surgery. The technique allows to determine in real time the position of a drill by tracking the position of either active or passive markers attacked to the drill. These markers are tracked by a stereoscopic optical camera. The marker is a light. The active system uses surgical drills with light emitting diodes, whilst in the passive system the light, emitted from a light source, is reflected back to the stereo cameras. The camera is a sensor connected with powerful computer which uses a dedicate software. The software allows the coordination of multiple images each other and to determine the location and orientation of the dental handpiece (drill) and the patient [3]. Usually, the display is a common monitor. The problem of the use of the monitor is that the operator needs to take his eyes off from the operation field to see the monitor, this problem can be solved by the use of smart glasses (SM). These glasses are wearable computing device, which comes with a head-mounted display. Using SM, surgeon intraoperatively can observe virtual images superimposed on operatory camp [4]. Thus, the patient becomes virtually transparent and the surgeon can visualize both structure and margins of a lesion that often are not directly visible to the naked eye. Today, the idea to use both virtual and augmented reality in oral surgery is not science fiction but

${ }^{\star}$ Correspondence to: Paolo Difalco, Via Pergusa,75 - 92020 - Palma di Montechiaro (Ag), Italy, E-mail: paolodifalco@tin.it

Received: August 13, 2019; Accepted: September 06, 2019; Published: September 09, 2019 
has become reality. It is our opinion that the development of virtual and augmented reality, will allow perform complex oral surgical procedures with more accuracy, speed, and safety.

\section{References}

1. Alur A, Shrivastav P, Jumde A (2014) Haptic technology: A comprehensive review of its applications and future prospects. IJCSIT 5: 6039-6043
2. Samadbeik M, Yaaghobi D, Bastani P, Abhari S, Rezaee R, et al. (2018) The applications of virtual reality technology in medical groups teaching. $J$ Adv Med Educ 6:123-129

3. Wang J, Suenaga H, Hoshi K, Yang L, Kobayashi E, et al. (2014) Augmented reality navigation with automatic marker-free image registration using 3-D image overlay for dental surgery. IEEE Transactions on Biomedical Engineering 61: 1295-1304

4. Badiali G, Ferrari V, Cutolo F, Freschi C, Caramella D, et al. (2014) Augmented reality as an aid in maxillofacial surgery: validation of a wearable system allowing maxillary repositioning. J Craniomaxillofac Surg 42:1970-1976.

Copyright: (C2019 Tortorici S. This is an open-access article distributed under the terms of the Creative Commons Attribution License, which permits unrestricted use, distribution, and reproduction in any medium, provided the original author and source are credited. 